\title{
A NOTE ON THICK SUBCATEGORIES OF STABLE DERIVED CATEGORIES
}

\author{
HENNING KRAUSE AND GREG STEVENSON
}

\begin{abstract}
For an exact category having enough projective objects, we establish a bijection between thick subcategories containing the projective objects and thick subcategories of the stable derived category. Using this bijection, we classify thick subcategories of finitely generated modules over strict local complete intersections and produce generators for the category of coherent sheaves on a separated Noetherian scheme with an ample family of line bundles.
\end{abstract}

\section{$\S 1$. Introduction}

Let $A$ be a not necessarily commutative ring, and let $\bmod A$ be the category of finitely presented $A$-modules. Consider the stable derived category of $A$ in the sense of Buchweitz [6], which is also called the triangulated category of singularities or just the singularity category, following work of Orlov [13]. This category is by definition the Verdier quotient of the bounded derived category of $\bmod A$ with respect to the triangulated subcategory consisting of all perfect complexes:

$$
\mathrm{D}^{b}(\bmod A) / \mathrm{D}^{b}(\operatorname{proj} A)
$$

In a number of recent articles, thick subcategories of this triangulated category have been studied and even classified, typically in terms of prime ideals of some appropriate cohomology ring (see [3], [4], [12], [16], [17]). In this note we point out a bijection between thick subcategories of the stable derived category and thick subcategories of the module category containing all projective modules. In a special case, this bijection has been observed by Takahashi [18]. Using our more general form of this bijection, we are able to extend [18, Theorem 4.6(1)] to complete intersections.

Further applications of this bijection arise from the study of generators of exact and triangulated categories. We illustrate this by results of Schoutens

Received November 24, 2011. Revised June 14, 2012. Accepted August 30, 2012.

First published online September 3, 2013.

2010 Mathematics Subject Classification. Primary 18E30; Secondary 13D09, 14 F05. 
[15, Theorem VI.8] and of Oppermann and Št'ovíček [12, Theorem 7]. In the first case, we prove a generation result for the category of coherent sheaves on a Noetherian separated divisorial scheme which, in the affine case, is contained in a result of Schoutens. In the latter case, we pose a question on the transfer of information from triangulated to exact categories.

\section{$\S 2$. Thick subcategories versus thick subcategories}

Let $A$ be an exact category in the sense of Quillen [14], and denote by $D^{b}(A)$ its bounded derived category (see [9], [19]). Suppose that A has enough projective objects. Let Proj $A$ be the full subcategory consisting of the projective objects, and view $D^{b}(\operatorname{Proj} A)$ as a thick subcategory of $D^{b}(A)$. The Verdier quotient

$$
\mathrm{D}^{b}(\mathrm{~A}) / \mathrm{D}^{b}(\operatorname{Proj} \mathrm{A})
$$

is by definition the stable derived category of A.

We are interested in thick subcategories of the stable derived category, and we observe that they correspond bijectively to thick subcategories of $\mathrm{D}^{b}(\mathrm{~A})$ containing all projective objects (see [19, Proposition II.2.3.1]). Recall that a full additive subcategory of a triangulated category is thick if it is closed under shifts, mapping cones, and direct summands.

A full additive subcategory $C$ of $A$ is called thick if it is closed under direct summands and has the following two out of three properties: for every exact sequence $0 \rightarrow X \rightarrow Y \rightarrow Z \rightarrow 0$ in A with two terms in $\mathrm{C}$, the third term belongs to $\mathrm{C}$ as well.

In the following, we identify $A$ with the full subcategory of $D^{b}(A)$ consisting of all complexes concentrated in degree 0 .

THEOREM 1. Let A be an exact category having enough projective objects. The map sending a subcategory $\mathrm{D}$ of $\mathrm{D}^{b}(\mathrm{~A})$ to $\mathrm{D} \cap \mathrm{A}$ induces a bijection between

(i) the thick subcategories of $\mathrm{D}^{b}(\mathrm{~A})$ containing all projective objects, and

(ii) the thick subcategories of $\mathrm{A}$ containing all projective objects.

The inverse map sends a thick subcategory $\mathrm{C}$ of $\mathrm{A}$ to the replete closure of $\mathrm{D}^{b}(\mathrm{C})$.

Proof. We may assume that $A$ is idempotent complete, keeping in mind that the idempotent completion of $D^{b}(A)$ is canonically equivalent to the bounded derived category of the idempotent completion of $A$ (see [1]). 
The first part of this proof is taken from [2, Appendix]. Suppose that $C$ is a thick subcategory of $A$ containing all projectives. The category $C$ inherits an exact structure from $A$, and the inclusion $C \rightarrow A$ induces therefore an exact functor $D^{b}(C) \rightarrow D^{b}(A)$. The fact that $C$ contains the projective objects implies that this functor is fully faithful (see, e.g., [19, Proposition III.2.4.1]). Thus, the full subcategory $\mathrm{D}$ of $\mathrm{D}^{b}(\mathrm{~A})$ consisting of objects isomorphic to a bounded complex of objects in $C$ is a thick subcategory. We claim that $\mathrm{C}=\mathrm{D} \cap \mathrm{A}$. Clearly, $\mathrm{C} \subseteq \mathrm{D} \cap \mathrm{A}$. Thus, we fix $X \in \mathrm{D} \cap \mathrm{A}$. Then $X$ is in $\mathrm{D}^{b}(\mathrm{~A})$ isomorphic to a bounded complex $C$ with differential $\delta$ such that $C^{n} \in \mathrm{C}$ for all $n$, and $C$ is acyclic in all degrees $n \neq 0$. Now we use that $C$ is thick. Thus, Coker $\delta^{r} \in \mathrm{C}$ for all $r \leq-2$, since $C^{n}=0$ for $n \ll 0$. Similarly, $\operatorname{Ker} \delta^{r} \in \mathrm{C}$ for all $r \geq 0$, since $C^{n}=0$ for $n \gg 0$. Moreover, we have an admissible monomorphism Coker $\delta^{-2} \rightarrow \operatorname{Ker} \delta^{0}$ such that the cokernel is isomorphic to $X$. We conclude that $X$ belongs to $\mathrm{C}$, and therefore $\mathrm{C}=\mathrm{D} \cap \mathrm{A}$.

Now fix a thick subcategory $D$ of $D^{b}(A)$ containing all projectives, and set $\mathrm{C}=\mathrm{D} \cap \mathrm{A}$. Each exact sequence $0 \rightarrow X \rightarrow Y \rightarrow Z \rightarrow 0$ in A gives rise to an exact triangle $X \rightarrow Y \rightarrow Z \rightarrow X[1]$ in $\mathrm{D}^{b}(\mathrm{~A})$. Thus, $\mathrm{C}$ is a thick subcategory of $A$. We claim that the functor $\mathrm{D}^{b}(\mathrm{C}) \rightarrow \mathrm{D}$ is an equivalence. In view of the first part of the proof, it suffices to show that each object $X$ in D is isomorphic to a complex of objects in C. We may assume that $X$ is a complex of projective objects with $X^{n}=0$ for $n \gg 0$ and $X$ acyclic in degrees $n \leq p$ for some integer $p$. Brutally truncating $X$ in degree $p$ gives an exact triangle $X^{\prime} \rightarrow X \rightarrow X^{\prime \prime} \rightarrow X^{\prime}[1]$ such that $X^{\prime}$ is a bounded complex of projective objects and $X^{\prime \prime}$ is acyclic in all degrees different from $p$. Clearly, $X^{\prime}$ belongs to $\mathrm{D}^{b}(\mathrm{C})$. By thickness of $\mathrm{D}$, the object $X^{\prime \prime}$ lies in $\mathrm{D}$ and thus is isomorphic to a shift of an object from C; since $X$ was acyclic below degree $p$, the brutal truncation $X^{\prime \prime}$ is a projective resolution of an object of $\mathrm{D} \cap \mathrm{A}$. It follows that $X$ is isomorphic to an object in $\mathrm{D}^{b}(\mathrm{C})$.

REMARK 2. As noted earlier, thick subcategories of the stable derived category correspond to thick subcategories of $D^{b}(A)$ containing the projective objects. It follows that the theorem gives a bijection between thick subcategories of the stable derived category and thick subcategories of $A$ containing all projective objects.

REMARK 3. In the theorem and its proof, one can replace the subcategory Proj A by any thick subcategory $\mathrm{P}$ of $\mathrm{A}$ having the property that each object $X \in \mathrm{A}$ admits an admissible epimorphism $P \rightarrow X$ with $P \in \mathrm{P}$. 
REMARK 4. A full additive subcategory $C$ of $A$ is thick and contains all projective objects if and only if it is closed under taking extensions, direct summands, cosyzygies, and syzygies.

\section{§3. Applications and comments}

In this section, we make some brief remarks concerning applications of the bijection of the last section. In particular, we connect the main result to work of Schoutens and Takahashi as promised in the introduction.

\subsection{Classification theorems}

We will explain how to use the main theorem to obtain two classification results, one new and one known, for thick subcategories of abelian categories.

We first use Theorem 1 to give a classification of the thick subcategories of $\bmod A$, which contain $A$, when $A$ is a strict local complete intersection ring; this extends $[18$, Theorem $4.6(1)]$. In order to state the classification, we need to introduce a hypersurface associated to $A$.

Let $A$ be a strict local complete intersection; that is, there is a regular local ring $B$ and a surjection $B \rightarrow A$ with kernel generated by a regular sequence. Set $\mathbb{X}=\operatorname{Spec} A, \mathbb{T}=\operatorname{Spec} B$, and $\mathcal{E}=\mathcal{O}_{\mathbb{T}}^{\oplus c}$, and let $\left\{b_{1}, \ldots, b_{c}\right\}$ form a regular sequence generating the kernel of $B \rightarrow A$. Let $\mathbb{Y}$ be the hypersurface defined by the section $\sum_{i=1}^{c} b_{i} x_{i}$ of $\mathcal{O}_{\mathbb{P}_{B}^{c-1}}(1)$, where the $x_{i}$ form a basis for the free $B$-module $H^{0}\left(\mathbb{P}_{B}^{c-1}, \mathcal{O}_{\mathbb{P}_{B}^{c-1}}(1)\right)$. In summary, we are concerned with the following commutative diagram:

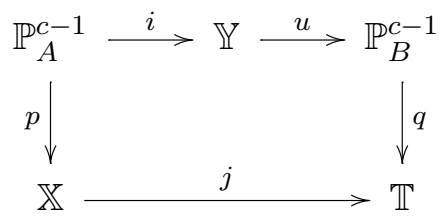

We will use Sing $\mathbb{Y}$ to denote the singular locus of $\mathbb{Y}$, that is, the set of $y \in \mathbb{Y}$ such that the local ring $\mathcal{O}_{\mathbb{Y}, y}$ is not regular.

The following corollary of Theorem 1, based upon [16, Corollary 10.5], shows that the hypersurface $\mathbb{Y}$ controls the thick subcategories of $\bmod A$ which contain the projectives. In the case that $A$ is a hypersurface, so that $\mathbb{Y} \cong \operatorname{Spec} A$, the same bijection has been proved by Takahashi in $[18$, Theorem 4.6(1)]. 
Corollary 5. Let $A$ be a strict local complete intersection, and let $\mathbb{Y}$ be the hypersurface as defined above. Then there is an order-preserving bijection

$$
\left\{\begin{array}{c}
\text { specialization closed } \\
\text { subsets of } \operatorname{Sing} \mathbb{Y}
\end{array}\right\} \longleftrightarrow\left\{\begin{array}{c}
\text { thick subcategories of } \\
\bmod A \text { containing } A
\end{array}\right\} .
$$

Proof. By Theorem 1, there is a bijection between thick subcategories of $\bmod A$ containing $A$ and thick subcategories of $\mathrm{D}^{b}(\bmod A)$ containing $A$. As stated in Remark 2 , the thick subcategories of $\mathrm{D}^{b}(\bmod A)$ containing $A$ are in bijection with the thick subcategories of the stable derived category $\mathrm{D}^{b}(\bmod A) / \mathrm{D}^{b}(\operatorname{proj} A)$. The result now follows from the classification of thick subcategories of the stable derived category given in [16, Corollary 10.5].

In a similar vein, we can apply the theorem to the work of Benson, Carlson, and Rickard [3] on stable categories in modular representation theory. Let $G$ be a finite group, and let $k$ be a field whose characteristic divides the order of $G$. We denote the category of finite-dimensional representations of $k G$ by $\bmod k G$, and we denote by stmod $k G$ its stable category which is obtained by annihilating all projective modules. We say that a subcategory $\mathrm{C}$ of $\bmod k G$ is a thick tensor ideal if it is a thick subcategory which is closed under tensoring with arbitrary objects of $\bmod k G$. Applying Theorem 1 to [3, Theorem 3.4] immediately recovers the following known result.

TheOREM 6. Let $k$ and $G$ be as above. Then there is an order-preserving bijection

$$
\left\{\begin{array}{c}
\text { specialization closed } \\
\text { subsets of } \operatorname{Proj} H^{*}(G, k)
\end{array}\right\} \longleftrightarrow\left\{\begin{array}{c}
\text { thick tensor ideals of } \\
\bmod k G \text { containing } k G
\end{array}\right\},
$$

where $H^{*}(G, k)$ denotes the cohomology of $G$ with coefficients in $k$.

Although this is implicit in the work of Benson, Carlson, and Rickard, it does not seem to be explicitly stated in the literature. One should also compare this with the analogue for the category of all $k G$-modules which appears in [4, Theorem 10.4].

\subsection{Generating categories of coherent sheaves on schemes}

We now use Theorem 1 to prove a generation result for categories of coherent sheaves on certain schemes, partially extending work of Schoutens in the affine case. In order to state the theorem, let us recall the notion of an ample family of line bundles. 
Let us fix a separated Noetherian scheme $\mathbb{X}$. A family of line bundles $\left\{\mathcal{L}_{i} \mid 1 \leq i \leq n\right\}$ on $\mathbb{X}$ is ample if there is a family $\left\{f_{\lambda} \mid \lambda \in \Lambda\right\}$ of sections $f_{\lambda} \in H^{0}\left(\mathbb{X}, \mathcal{L}_{i_{\lambda}}^{\otimes m_{\lambda}}\right)$ with $1 \leq i_{\lambda} \leq n$ and $m_{\lambda}>0$ such that the $\mathbb{X}_{f_{\lambda}}=\{x \in$ $\left.\mathbb{X} \mid\left(f_{\lambda}\right)_{x} \notin \mathfrak{m}_{x} \mathcal{L}_{x}^{\otimes m}\right\}$, where $\mathfrak{m}_{x}$ is the maximal ideal of $\mathcal{O}_{\mathbb{X}, x}$, form an open affine cover of $\mathbb{X}$. Of course, one can just keep in mind the example of an affine scheme $\mathbb{X}=\operatorname{Spec} A$ and take $\left\{\mathcal{O}_{\mathbb{X}}\right\}$ for the ample family. We denote by coh $\mathbb{X}$ the abelian category of coherent sheaves of $\mathcal{O}_{\mathbb{X}}$-modules. This section is dedicated to the proof of the following theorem.

TheOREM 7. Let $\mathbb{X}$ be a separated Noetherian scheme, and let $\left\{\mathcal{L}_{i} \mid 1 \leq\right.$ $i \leq n\}$ be an ample family of line bundles on $\mathbb{X}$. Then the smallest thick subcategory of coh $\mathbb{X}$ containing the set of coherent $\mathcal{O}_{\mathbb{X}}$-modules

$$
\mathcal{S}=\left\{\mathcal{L}_{i}^{\otimes m} \otimes \mathcal{O}_{\mathbb{X}} \mathcal{O}_{\mathcal{V}(x)}^{\alpha} \mid 1 \leq i \leq n, m \in \mathbb{Z}, x \in \operatorname{Sing} \mathbb{X}, \alpha \in\{0,1\}\right\},
$$

where $\mathcal{O}_{\mathcal{V}(x)}$ denotes the structure sheaf of the closed subset $\mathcal{V}(x)$ endowed with the reduced induced scheme structure and $\mathcal{O}_{\mathcal{V}(x)}^{0}=\mathcal{O}_{\mathbb{X}}$, is the whole category of coherent $\mathcal{O}_{\mathbb{X}}$-modules.

The proof passes through the homotopy category of injective sheaves of $\mathcal{O}_{\mathbb{X}}$-modules. Let $\mathrm{K}(\mathbb{X})$ denote the homotopy category of complexes of quasicoherent $\mathcal{O}_{\mathbb{X}}$-modules, and let $\mathrm{K}(\operatorname{Inj} \mathbb{X})$ be the full subcategory consisting of complexes of injective quasicoherent $\mathcal{O}_{\mathbb{X}}$-modules. We identify each quasicoherent sheaf with the corresponding complex in $\mathrm{K}(\mathbb{X})$ concentrated in degree 0 .

Lemma 8. Let $\mathbb{X}$ be a Noetherian scheme as above, and let $\mathrm{C}$ be a subcategory of coh $\mathbb{X}$ containing the sheaves $\left\{\mathcal{L}_{i}^{\otimes m} \mid 1 \leq i \leq n, m \in \mathbb{Z}\right\}$. Suppose that any complex $Y$ of injective quasicoherent $\mathcal{O}_{\mathbb{X}}$-modules is null-homotopic provided that

$$
\operatorname{Hom}_{\mathrm{K}(\mathbb{X})}(X, Y[n])=0 \quad \text { for all } X \in \mathrm{C}, n \in \mathbb{Z}
$$

Then the smallest thick subcategory of coherent sheaves containing $\mathrm{C}$ is $\operatorname{coh} \mathbb{X}$.

Proof. The functor $\mathbf{i}: \operatorname{coh} \mathbb{X} \rightarrow \mathrm{K}(\operatorname{Inj} \mathbb{X})$ taking a sheaf to its injective resolution extends to an equivalence $\mathrm{D}^{b}\left(\operatorname{coh} \mathcal{O}_{\mathbb{X}}\right) \stackrel{\sim}{\rightarrow} \mathrm{K}(\operatorname{Inj} \mathbb{X})^{c}$ onto the full subcategory of compact objects of $\mathrm{K}(\operatorname{Inj} \mathbb{X})$, by [8, Proposition 2.3]. Note that

$$
\operatorname{Hom}_{\mathrm{K}(\mathbb{X})}(\mathbf{i} X, Y) \stackrel{\sim}{\rightarrow} \operatorname{Hom}_{\mathrm{K}(\mathbb{X})}(X, Y) \quad \text { for all } Y \in \mathrm{K}(\operatorname{Inj} \mathbb{X}),
$$


by [8, Lemma 2.1]. The assumption on $C$ implies that the thick subcategory of $\mathrm{K}(\operatorname{Inj} \mathbb{X})$ generated by $\mathbf{i}(C)$ equals $\mathrm{K}(\operatorname{Inj} \mathbb{X})^{c}$. This follows from a standard argument involving Bousfield localization (see [10, Lemma 2.2]). Thus, the correspondence in Theorem 1, together with Remark 3, implies that C generates $\operatorname{coh} \mathbb{X}$.

While it is possible to give a "global" proof of the theorem, it seems worthwhile to pass through the affine case; this lightens the technical load and makes the comparison with Schoutens's work clearer.

Proposition $9\left([15 \text {, Theorem VI.8 }]^{*}\right)$. Let $A$ be a commutative Noetherian ring. Then the smallest thick subcategory of the category of A-modules containing $A$ and $A / \mathfrak{p}$ for all $\mathfrak{p}$ in the singular locus of $A$ equals the category of Noetherian A-modules.

Proof. In view of Lemma 8, it suffices to show that each complex $X$ of injective $A$-modules is null-homotopic provided that

$$
\operatorname{Hom}_{\mathrm{K}(A)}(A, X[n])=0 \quad \text { and } \quad \operatorname{Hom}_{\mathrm{K}(A)}(A / \mathfrak{p}, X[n])=0
$$

for all $n \in \mathbb{Z}$ and $\mathfrak{p} \in \operatorname{Sing} A$.

Thus, we fix a complex $X$ satisfying these conditions. The first condition implies that $X$ is acyclic. We may assume that $X$ is homotopically minimal, that is, that there is no nonzero direct summand of $X$ which is null-homotopic (see [8, Appendix B]). This means that for each $n \in \mathbb{Z}$ the truncated complex

$$
X^{n} \rightarrow X^{n+1} \rightarrow X^{n+2} \rightarrow \cdots
$$

yields a minimal injective resolution of $Z^{n}(X)$. Localizing at a prime ideal $\mathfrak{p}$ preserves this property. If $\mathfrak{p} \notin \operatorname{Sing} A$, then $Z^{n}(X)_{\mathfrak{p}}$ is injective, and therefore $Z^{n+1}(X)_{\mathfrak{p}}=0$. Thus, $X_{\mathfrak{p}}=0$. Now consider for each $n \in \mathbb{Z}$ the exact sequence

$$
0 \rightarrow Z^{n}(X) \rightarrow X^{n} \rightarrow Z^{n+1}(X) \rightarrow 0
$$

of modules supported on $\operatorname{Sing} A$. If this sequence does not split, one finds a finitely generated submodule $U$ of $Z^{n+1}(X)$ isomorphic to $A / \mathfrak{p}$ for some $\mathfrak{p} \in \operatorname{Sing} A$ such that $\operatorname{Ext}_{A}^{1}\left(U, Z^{n}(X)\right) \neq 0$. This follows from Baer's criterion,

*It should be noted that the result we state here is weaker than Schoutens's [15, Theorem VI.8] in the sense that his result uses only cosyzygies (it does not require syzygies) and gives a bound on the required number of steps to generate. 
and one uses that the prime ideals in $\operatorname{Sing} A$ form a specialization closed subset of $\operatorname{Spec} A$. Now observe that

$$
\operatorname{Hom}_{\mathrm{K}(A)}(-, X[n+1]) \cong \operatorname{Ext}_{\mathrm{A}}^{1}\left(-, Z^{n}(X)\right)
$$

Thus, the assumption on $X$ implies that it is null-homotopic.

With the affine case in hand, we can now complete the proof of the theorem.

Proof of Theorem \%. We proceed essentially as in the affine case; that is, we show that if $X$ is a complex in $\mathrm{K}(\operatorname{Inj} \mathbb{X})$ which is not null-homotopic, then some object of $\mathcal{S}$ maps to a suspension of $X$. So let us fix such a complex $X$. We may assume that $X$ is acyclic as the tensor powers of the sheaves in the ample family generate the derived category (see [11, Example 1.11]) and thus detect any complex having nonzero cohomology.

Since $X$ is not null-homotopic, there exists a $j \in \mathbb{Z}$ such that $Z^{j}(X)$ is not injective. Let $f \in H^{0}\left(\mathbb{X}, \mathcal{L}_{i}^{\otimes m}\right)$ be a section such that $\mathbb{X}_{f}=\left\{x \in \mathbb{X} \mid f_{x} \notin\right.$ $\left.\mathfrak{m}_{x} \mathcal{L}_{x}^{\otimes m}\right\}$ is an open affine subscheme on which $\left.Z^{j}(X)\right|_{\mathbb{X}_{f}}$ is not injective; we can find such an $f$ by ampleness of the family of line bundles. In particular, $\left.X\right|_{\mathbb{X}_{f}}$ is nonzero in $\mathrm{K}\left(\operatorname{Inj} \mathbb{X}_{f}\right)$. Hence, by the proof of the theorem, there is an $x \in \operatorname{Sing} \mathbb{X}$ and a nonzero map $g \in \operatorname{Hom}_{\mathrm{K}_{\left(\mathbb{X}_{f}\right)}}\left(\left.\mathcal{O}_{\mathcal{V}(x)}\right|_{\mathbb{X}_{f}},\left.X\right|_{\mathbb{X}_{f}}[j+1]\right)$ for some integer $j$. By [7, Lemma III.5.14] there exists an $m^{\prime} \geq 0$ so that $f^{m^{\prime}} g$ lifts to a morphism

$$
\tilde{g}: \mathcal{O}_{\mathcal{V}(x)} \rightarrow X \otimes \mathcal{O}_{\mathbb{X}} \mathcal{L}_{i}^{\otimes m^{\prime}}[j+1]
$$

The map $\tilde{g}$ is not null-homotopic because if it were this would yield a nullhomotopy for $f^{m^{\prime}} g$ and thus $g$. It just remains to note that, by adjunction, the map $\tilde{g}$ is equivalent to a nonzero morphism $\mathcal{O}_{\mathcal{V}(x)} \otimes \mathcal{O}_{\mathbb{X}} \mathcal{L}_{i}^{\otimes-m^{\prime}} \rightarrow X[j+1]$ witnessing the fact that $X$ is not null-homotopic.

\subsection{Strong generators}

We end this note by asking for some further transfer from triangulated to exact categories. Strong generators of triangulated categories were introduced by Bondal and van den Bergh [5, Section 2.2]. There seems to be no obvious analogue for exact categories. So it would be interesting to translate the following prototypical result from a special version of [12, Theorem 7] into a statement about abelian categories, using the bijection from Theorem 1 . 
THEOREM 10. Let $k$ be a field, and let A be a $k$-linear abelian category which is Hom-finite and has enough projective objects. If $\mathrm{D}$ is a thick subcategory of $\mathrm{D}^{b}(\mathrm{~A})$ which contains all projective objects and admits a strong generator, then $\mathrm{D}=\mathrm{D}^{b}(\mathrm{~A})$.

Acknowledgment. It is a pleasure to thank the referees for helpful comments regarding the presentation of this material.

\section{REFERENCES}

[1] P. Balmer and M. Schlichting, Idempotent completion of triangulated categories, J. Algebra 236 (2001), 819-834. MR 1813503. DOI 10.1006/jabr.2000.8529.

[2] A. Beligiannis and H. Krause, Thick subcategories and virtually Gorenstein algebras, Illinois J. Math. 52 (2008), 551-562. MR 2524651.

[3] D. J. Benson, J. F. Carlson, and J. Rickard, Thick subcategories of the stable module category, Fund. Math. 153 (1997), 59-80. MR 1450996.

[4] D. J. Benson, S. B. Iyengar, and H. Krause, Stratifying modular representations of finite groups, Ann. of Math. (2) 174 (2011), 1643-1684. MR 2846489. DOI 10.4007/ annals.2011.174.3.6.

[5] A. Bondal and M. van den Bergh, Generators and representability of functors in commutative and noncommutative geometry, Mosc. Math. J. 3 (2003), 1-36, 258. MR 1996800.

[6] R.-O. Buchweitz, Maximal Cohen-Macaulay modules and Tate cohomology over Gorenstein rings, preprint, 1987.

[7] R. Hartshorne, Algebraic Geometry, Grad. Texts in Math. 52, Springer, New York, 1977. MR 0463157.

[8] H. Krause, The stable derived category of a Noetherian scheme, Compos. Math. 141 (2005), 1128-1162. MR 2157133. DOI 10.1112/S0010437X05001375.

[9] A. Neeman, The derived category of an exact category, J. Algebra 135 (1990), 388394. MR 1080854. DOI 10.1016/0021-8693(90)90296-Z.

[10] - The connection between the $K$-theory localization theorem of Thomason, Trobaugh and Yao and the smashing subcategories of Bousfield and Ravenel, Ann. Sci. Éc. Norm. Supér. (4) 25 (1992), 547-566. MR 1191736.

[11] - The Grothendieck duality theorem via Bousfield's techniques and Brown representability, J. Amer. Math. Soc. 9 (1996), 205-236. MR 1308405. DOI 10.1090/ S0894-0347-96-00174-9.

[12] S. Oppermann and J. Št'ovíček, Generating the bounded derived category and perfect ghosts, Bull. Lond. Math. Soc. 44 (2012), 285-298. MR 2914607. DOI 10.1112/blms/ bdr093.

[13] D. O. Orlov, Triangulated categories of singularities and D-branes in LandauGinzburg models (in Russian), Tr. Mat. Inst. Steklova 246 (2004), Algebr. Geom. Metody, Svyazi i Prilozh., 240-262; English translation in Proc. Steklov Inst. Math. 246 (2004), 227-248. MR 2101296.

[14] D. Quillen, "Higher algebraic K-theory, I" in Algebraic K-Theory, I: Higher $K$ Theories (Seattle, 1972), Lecture Notes in Math. 341, Springer, Berlin, 1973, 85-147. MR 0338129. 
[15] H. Schoutens, Projective dimension and the singular locus, Comm. Algebra 31 (2003), 217-239. MR 1969220. DOI 10.1081/AGB-120016756.

[16] G. Stevenson, Subcategories of singularity categories via tensor actions, preprint, arXiv:1105.4698v3 [math.AG]

[17] R. Takahashi, Classifying thick subcategories of the stable category of CohenMacaulay modules, Adv. Math. 225 (2010), 2076-2116. MR 2680200. DOI 10.1016/ j.aim.2010.04.009.

[18] - Thick subcategories over Gorenstein local rings that are locally hypersurfaces on the punctured spectra, J. Math. Soc. Japan 65 (2013), 357-374.

[19] J.-L. Verdier, Des catégories dérivées des catégories abéliennes, Astérisque 239, Soc. Math. France, Paris, 1997. MR 1453167.

Henning Krause

Fakultät für Mathematik

Universität Bielefeld

D-33501 Bielefeld

Germany

hkrause@math.uni-bielefeld.de

Greg Stevenson

Fakultät für Mathematik

Universität Bielefeld

D-33501 Bielefeld

Germany

gstevens@math.uni-bielefeld.de 